\title{
Propuesta de control de posición y velocidad de motores en paralelo implementando PAC y HMI para una grúa tipo pórtico
}

\section{Proposal for position and speed control of parallel motors by implementing PAC and HMI in a gantry-type crane}

\begin{abstract}
E. Flores-García ${ }^{a}$, J.C. Quezada-Quezada ${ }^{b}$, R.V.H. Calderón-Medina ${ }^{c}$, A. Pérez-Pérez ${ }^{d}$ Abstract:

Engineering creates and employs a large amount of machinery and instruments helping humans to perform tasks with some ease; an example of that are cranes, which enable to move loads of huge weights without the necessity of human force. Parallel motors control is considered a favourable strategy to implement in cranes, for example at port industry, due to the complexity of this type of systems. This work constitutes a terminal academic project of practical application, focused on the industrial-type processes automation, in which a proposal for position and speed control of alternating current parallel motors is stated in order to actuate a gantry-type crane, by using automation technologies such as a GE Fanuc PAC System RX3i, whose programming is accomplished by means of LD language through the software Proficy Machine Edition; an HMI, developed using the software Cimplicity Workbench; and the communication protocol MODBUS, for communication between motors and variable frequency drives for speed control.
\end{abstract}

Keywords:

Gantry crane, HMI, motor control, PAC, variable frequency drive

Resumen:

La ingeniería crea y emplea una gran cantidad de maquinaria e instrumentos que ayudan al ser humano a realizar tareas con cierta facilidad; un ejemplo son las grúas, las cuales posibilitan mover cargas de enorme peso sin la necesidad de la fuerza humana. El control de motores en paralelo se considera una estrategia favorable para implementar en grúas, por ejemplo, en la industria portuaria, debido a la complejidad de este tipo de sistemas. El presente trabajo constituye un proyecto académico terminal de aplicación práctica, con enfoque a la automatización de procesos de tipo industrial, en el cual se plantea una propuesta de control de posición y velocidad de motores de corriente alterna en paralelo para la actuación de una grúa tipo pórtico, usando tecnologías de automatización tales como un PAC System RX3i de GE Fanuc, cuya programación se realiza en lenguaje LD mediante el software Proficy Machine Edition; una HMI, desarrollada mediante el software Cimplicity Workbench; y el protocolo de comunicación MODBUS, para la comunicación entre los motores y los variadores de frecuencia para el control de velocidad.

\section{Palabras Clave:}

Control de motores, grúa pórtico, HMI, PAC, variador de frecuencia

\section{Introducción}

El desarrollo de maquinaria y tecnología para las industrias es cada vez mayor y más sofisticado, debido a la tendencia de éstas a su modernización para garantizar su competitividad, demandando mejoras para los procesos en cuanto a eficiencia, tiempos, seguridad, productividad, etc.; la industria portuaria no es la excepción, es una de las más activas ya que bajo ésta se realiza el $90 \%$ del comercio con otros países en un canal bidireccional: exportaciones e importaciones. [1] La manipulación de mercancías en los puertos sería prácticamente imposible sin el uso de grúas, cuya actuación se produce comúnmente utilizando motores

\footnotetext{
a Autor de Correspondencia, Universidad Autónoma del Estado de Hidalgo, Escuela Superior de Tizayuca, https://orcid.org/0000-00024606-8217, email: efloresg@uaeh.edu.mx

b Universidad Autónoma del Estado de Hidalgo, https://orcid.org/0000-0003-3125-3708, email: jcarlos@uaeh.edu.mx

c Universidad Autónoma del Estado de Hidalgo, https://orcid.org/0000-0002-7292-6051, email: rafael_calderon@uaeh.edu.mx

${ }^{d}$ Universidad Autónoma del Estado de Hidalgo, https://orcid.org/0000-0002-4824-3357, email: pe359716@uaeh.edu.mx
} 
eléctricos, generalmente de corriente alterna. El control de motores dispuestos en paralelo permite un mejor desempeño de ese tipo de sistemas de carga, generando un menor consumo de energía lato sensu y mayor rendimiento, y consecuentemente un incremento en las ganancias, algo de gran interés para las industrias.

En este trabajo, se presenta una propuesta de control de posición y velocidad de motores en paralelo implementado mediante un Controlador de Automatización Programable (PAC, por sus siglas en Inglés de Programmable Automation Controller), cuya lógica de programación se desarrolla en el lenguaje Diagrama Escalera (LD, por sus siglas en Inglés de Ladder Diagram), uno de los cinco lenguajes de programación para controladores programables normados por la International Electrotechnical Commission a través del estándar IEC 61131-3.

La lógica de control, grabada en la memoria del PAC, ejecuta las instrucciones de salida (señales de control) hacia los actuadores con respecto a las entradas recibidas (variables medidas) por parte de los sensores de posición, ubicados en puntos estratégicos de la estructura de la grúa.

\section{Evaluación de la propuesta de automatización}

La tecnología actual determina que el costo para la empresa de mover una tonelada de carga en un minuto es igual a 14.28 dólares, y la empresa cobra 57.19 dólares. Cada minuto se mueven alrededor de 11 toneladas y se puede trabajar hasta 24 horas con 6 operadores (4 horas por operador); si se toma un flujo medio de 12 horas (3 operadores) de trabajo por día, al año se habrán trabajado 4,380 h y se habrán movido $2,890,800$ toneladas al año.

Al realizar una evaluación técnica de proyecto a través del curso curricular antecedente Formulación y Evaluación de Proyectos, se ha estimado que, con la tecnología propuesta, la reducción del consumo eléctrico puede ser de un 10 a un $15 \%$, y un aumento en la eficiencia de movimiento para una manipulación de carga de 11.5 toneladas por minuto, lo cual genera un menor costo de operación y consecuentemente una mayor ganancia producida por esos movimientos.

La tecnología actual señala el uso de los motores de forma constante y sin conmutación entre éstos; por lo que, al operar los motores en paralelo, el ahorro en el proceso puede verse reflejado en un costo por tonelada de 14.18 dólares con el movimiento de 11.5 toneladas por minuto. Si se realiza la comparación, se obtiene que, si se toma un flujo medio de 12 horas (3 operadores) de trabajo por día, al año se habrán trabajado 4,380 h y se habrán movido 3,022,200 toneladas al año.

\begin{tabular}{cccc}
\hline Tecnología Tn/min & $\begin{array}{c}\text { Costo de } \\
\text { operación por } \\
\text { tonelada } \\
\text { (dólares, USD) }\end{array}$ & $\begin{array}{c}\text { Precio por } \\
\text { tonelada } \\
\text { (USD) }\end{array}$ \\
\hline Actual & 11 & 14.28 & 57.19 \\
\hline Propuesta & 11.5 & 14.18 & 57.19
\end{tabular}

Tabla 1. Cifras por manipulación de carga en función de la tecnología empleada.

\begin{tabular}{cccc}
\hline $\begin{array}{c}\text { Horas de } \\
\text { Toneladas operación al } \\
\text { año }\end{array}$ & $\begin{array}{c}\text { Costo de } \\
\text { operación } \\
\text { total (USD) }\end{array}$ & $\begin{array}{c}\text { Precio } \\
\text { total (USD) }\end{array}$ \\
\hline $1,511,100$ & 2,190 & $21,427,398$ & $86,419,809$
\end{tabular}

Tabla 2. Cifras anuales por manipulación de carga en intervalo de seis horas de operación.

A partir de las cifras mostradas en la Tabla 2, basadas en la Tabla 1, se puede apreciar que la ganancia total por la operación anual es de 64,992,411 USD; y que el incremento en la ganancia anual con respecto a la tecnología actual $(62,022,114)$ sería de 2,970,297 USD.

\section{Control de motores en paralelo en grúas y Protocolos de comunicación}

El control de motores en paralelo es un tipo de control implementado comúnmente en sistemas donde los motores desempeñan en momentos diferentes acciones y en algunos otros las mismas, con lo que se obtiene una optimización de los movimientos y un mejor rendimiento en diferentes aspectos, por ejemplo, del control mismo y en el consumo eléctrico.

El control de una grúa pórtico representa un desafío para los ingenieros de control, debido a que es un sistema complejo, y multivariable. Normalmente, aplicando técnicas clásicas de control se obtienen respuestas favorables que realizan satisfactoriamente su trabajo.

Desde sus inicios y hasta hoy en día, la automatización ha impactado en todos los aspectos y sectores de la sociedad en general, siempre con el objetivo de proporcionar mayor productividad, facilidad, eficiencia, seguridad, comodidad, y optimización de recursos tanto humanos como materiales [2].

El concepto de automatización se concretó con la aparición de los autómatas programables, permitiendo 
mayor eficiencia en los procesos industriales. Hoy en día, el tema de la mejora de los procesos automatizados atraviesa por el desarrollo y la implementación de redes de comunicación.

La Organización Internacional de Estandarización (ISO), creó en 1984 el comité de la Interconexión de Sistemas Abiertos (OSI, por sus siglas en Inglés de Open System Interconnection), para el desarrollo de estándares de comunicación que permitieran la interoperabilidad entre equipos de distintos fabricantes. El modelo OSI se caracteriza por dividir las comunicaciones en siete niveles o capas, cada una de las cuales comprende funciones específicas. Otra caracterización del modelo es la división de los siete niveles en dos grupos; el primero toma los niveles 1 al 4 para los servicios de transporte de información, y el segundo incluye los niveles restantes, 5 al 7 , para servicios de soporte al usuario [3].

Los protocolos de comunicación se pueden definir como las normas y/o reglas a las que se deben ajustar dos 0 más equipos que se deseen comunicar. Éstos pueden ser ya sea propietarios ó abiertos; un protocolo propietario pertenece a una sola firma (marca, compañía) y para ser empleado es necesario contar con una licencia concedida por la empresa. En cambio, uno abierto es lo contrario, debido a que es público y de fácil y libre acceso.

La comunicación basada en señales analógicas usa rangos establecidos de voltaje ó corriente para la interacción entre equipos, empleando una red punto a punto; mientras que la comunicación basada en señales digitales emplea protocolos mediante el uso de buses de campo en una red multipunto [4].
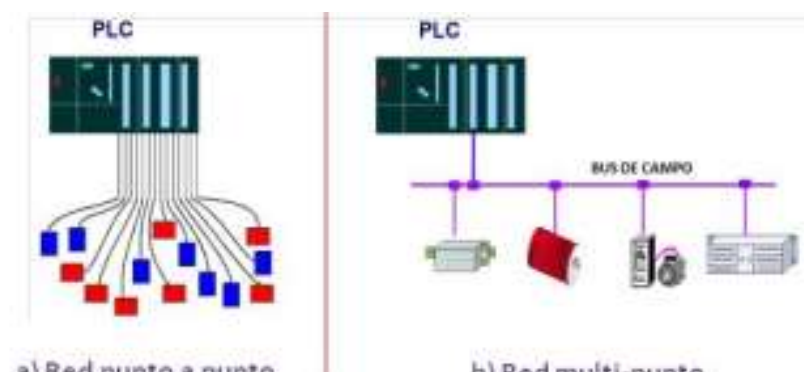

b) Red multi-punto

Fig. 1. Comparación de redes usadas en comunicación analógica (a) y comunicación digital (b)

Un bus de campo es un sistema de transmisión de información bidireccional, empleado en dispositivos de campo (sensores, actuadores) y dispositivos de control, con el objetivo de remplazar la comunicación punto a punto [1]. El uso de buses de campo reduce costos de instalación y mantenimiento, y mejora el funcionamiento del sistema, debido a que requiere un solo cable para diversos nodos [5].

Los buses de campo permiten que los protocolos de comunicación cubran frecuentemente tres niveles del modelo OSI: Nivel físico, que consta del medio físico de conexión y la naturaleza de la señal, entre otros; Nivel de enlace, las funciones y los servicios de red para el intercambio de información; y Nivel de aplicación, donde se define el significado de los datos, es una capa dirigida al usuario.

El uso de las comunicaciones y de la tecnología van de la mano al hablar de automatización de procesos industriales. Actualmente es difícil encontrar industrias consolidadas que no tengan sus procesos automatizados a niveles considerables. Es decir, procesos en los cuales se desarrolla una gran productividad con cada vez menor intervención humana, por distintas causas como seguridad, precisión y tiempos de producción, por mencionar algunos aspectos.

La automatización en una empresa o industria puede considerar desde la producción hasta la comodidad de los trabajadores. Desde el ingreso de la materia prima al proceso, hasta el almacenamiento del producto para la espera de su distribución. Una de las máquinas que más intervienen para el traslado de material y/o producto empleada en este último punto, es la grúa tipo puente o grúa viajera, ya sea para almacenar el producto fabricado o para introducir cierta materia a un proceso.

La globalización junto con el desarrollo de la tecnología, han transformado los criterios de producción, reestructurando los sistemas de distribución; demandando sistemas que mejoren la calidad del control, manipulación, y distribución de productos.

\section{MODBUS}

Uno de los protocolos de comunicación abiertos más utilizados por sus características es el MODBUS, basado en una topología de bus lineal cuyo propósito es el intercambio de información entre varios dispositivos a través de un mismo bus que se compone de un elemento principal, único en tomar la iniciativa para el intercambio de información, y uno o varios elementos subordinados, que no pueden iniciar comunicación directamente.

En el mundo de la automatización el control se divide en dos partes; el control de procesos, y el control de máquinas. En este último es más importante la ejecución en tiempo real, debido a que el controlador debe 
reaccionar a eventos de entrada. Uno de estos controladores es el PLC, que puede reaccionar mediante salidas digitales y analógicas a eventos recibidos mediante entradas digitales y analógicas. Además de que puede contar con buses de comunicación como PROFIBUS DP o MODBUS, y ser incluido de esta forma en sistemas SCADA. Este tipo de equipos junto con los PLC demuestran ser eficientes y confiables en procesos secuenciales. Es por eso que en el presente trabajo se implementa este tipo de protocolo de comunicación sofisticado para la interacción del PAC y el variador de velocidad, que de acuerdo con sus características y propiedades son equipos que pueden generar un sistema de control más robusto para el traslado de materia prima o productos dentro de un almacén o un proceso industrial.

\section{Encoders}

En la automatización, los encoders o codificadores se utilizan como sensores de cantidades angulares de posición, velocidad y aceleración. También permiten medir el movimiento lineal apoyándose de husillos, cremalleras, ruedas de medición o cables extensibles.

Los encoders convierten una entrada mecánica en señales eléctricas, que pueden procesarse mediante contadores, tacómetros, PLCs, y computadoras industriales [10].

Las industrias tienden a utilizar habitualmente los potenciómetros más que cualquier otro dispositivo para medir el ángulo. No obstante, durante los últimos 25 años, el uso de técnicas sin contacto ha crecido significativamente. La tendencia continua hacia las mediciones sin contacto se debe a los problemas de desgaste y fiabilidad de los potenciómetros, especialmente en entornos complicados, por ejemplo de vibración importante, o durante grandes periodos de tiempo [6].

Los codificadores ópticos son un tipo común de codificador rotatorio sin contacto; funcionan haciendo incidir una luz a través de, o dentro de, una rejilla óptica y calculando la posición angular por la intensidad de la luz que vuelve. La mayoría de los dispositivos ópticos son incrementales; normalmente, la información de posición se proporciona mediante una serie de pulsos, sobre todo en la fase de cuadratura, de forma que se pueda determinar el sentido del desplazamiento; se les suele denominar pulsos A/B. Un tren de pulsos separado, denominado normalmente referencia $Z$, proporciona un pulso por revolución para actuar como un dato o marca de referencia.

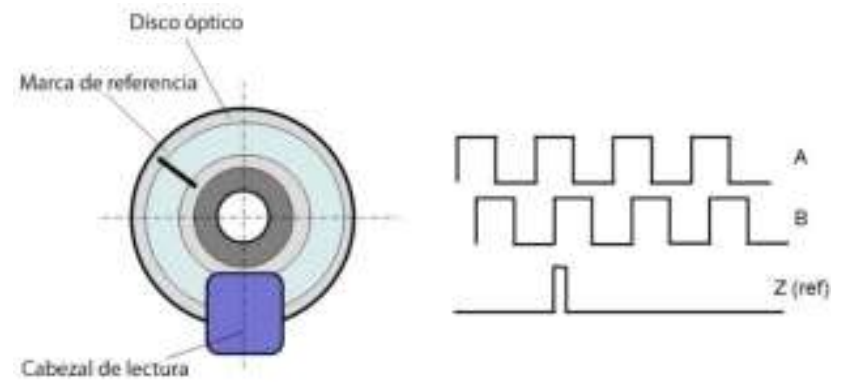

Fig. 2. Representación esquemática de codificador óptico incremental y su tren de pulsos.

El codificador absoluto es similar, utilizando un tipo de escala diferente, lo que permite determinar el ángulo absoluto con solo encenderse, sin necesidad de ninguna marca de referencia; normalmente, este tipo de codificadores tienen una salida digital y su resolución se define por el número de bits de salida. Un dispositivo de 10 bits ofrece 1.024 recuentos; uno de 11 bits ofrece 2.048 recuentos, y así sucesivamente.

\section{Motores de corriente alterna}

Son máquinas eléctricas rotativas, capaces de convertir la energía eléctrica trifásica suministrada como alimentación, en energía mecánica. La energía eléctrica origina campos magnéticos rotativos en el bobinado del estator, lo que provoca que el arranque de estos motores no necesite circuito auxiliar; son más pequeños y livianos que uno monofásico de inducción de la misma potencia, debido a eso su fabricación representa un costo menor.

Los motores eléctricos trifásicos se fabrican en las más diversas potencias, desde una fracción de caballo de potencia (HP) hasta varios miles de caballos; asimismo, se construyen para prácticamente todas las tensiones y frecuencias normalizadas (50 y $60 \mathrm{~Hz}$ ), y muy a menudo están equipados para operar a dos tensiones nominales distintas.

\section{Control PID}

El PID es un elemento clásico de control que está compuesto por tres partes o acciones de control; Proporcional, Integral, y Derivativa. No es obligatorio que se presenten las tres juntas en todos los controladores, por ello se pueden tener controladores de tipo PI o PD. Constituye un elemento de control sencillo y fácil de construir y calcular; ofrece muy buenos resultados en la corrección de señales en tiempo real, ya que es relativo a la realimentación del sistema; se considera muy estable y no requiere conocimiento del funcionamiento interno del sistema a controlar. En el software Proficy Machine Edition se expresa como muestra la Fig. 3. 


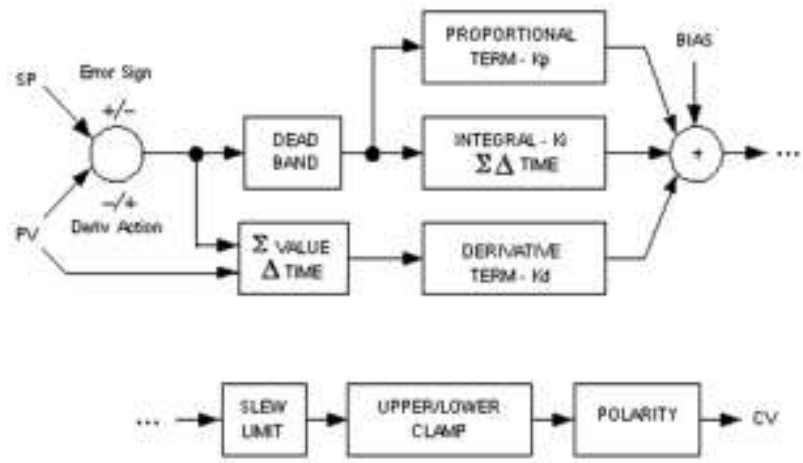

Fig. 3. Representación del PDI en Proficy Machine Edition.

Su forma en bloque de control en lenguaje de programación LD para PLC/PAC se presenta en la Fig. 4.

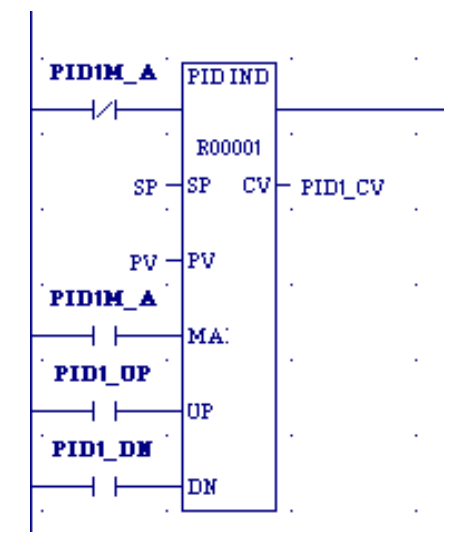

Fig. 4. Bloque de control PID en lenguaje Diagrama Escalera.

La utilización de este tipo de control en grúas ayuda a disminuir "tirones" u oscilaciones en el sistema, contribuyendo a evitar accidentes por sobretensión o forzamiento de las líneas que conectan a los componentes.

\section{Propuesta planteada}

Para los fines de este trabajo, se propone realizar el control PID simple, considerando la velocidad y la posición lineal del motor; se utiliza un PAC System RX3i de GE Fanuc con un CPU 310, un módulo de entradas IC693MDL634 [7], dos módulos de alta velocidad HSC304 (8 entradas y 7 salidas) con cuatro contadores cada uno, un módulo de comunicación Ethernet ETM001 [3], un Quick panel de 6 pulgadas (control y visualización), dos encoder tipo incremental, cuatro sensores de límite, y dos motores de corriente alterna; además del software Proficy Machine Edition para realizar la programación del PAC mediante el lenguaje Diagrama Escalera, así como el desarrollo de la HMI en el quick panel y en la PC mediante Cimplicity, para monitoreo y visualización de forma remota.

\section{Descripción del sistema}

En el sistema propuesto se considera el uso de un joystick de 0.5 VDC conectado a una entrada analógica que, con la variación de sus movimientos en los ejes $X$ (horizontal) y $Y$ (vertical) permita manipular la velocidad y posición del actuador final que será controlado por variadores de frecuencia AF-60 de GE Fanuc; la información se trasmitirá a través del protocolo MODBUS, que además de ser emisor recibirá la señal de retroalimentación mediante el protocolo proveniente del driver encontrado en el registro 1600 , y al mismo tiempo, por medio de un encoder, se retroalimentará la señal de posición del actuador final, retrasmitiendo la información al otro driver en espera de su funcionamiento basado en casos de actuación de los motores.

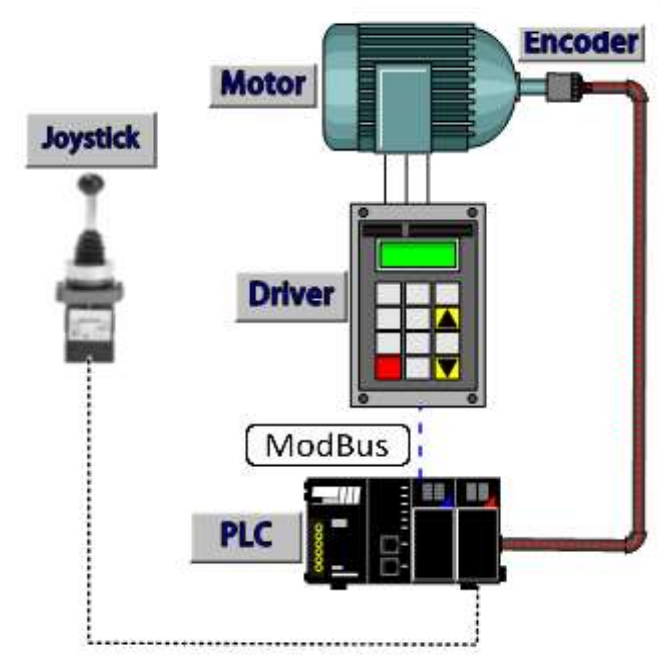

Fig. 5. Interrelación de componentes del sistema.

El módulo IC695HSC304 permite procesar señales digitales de entrada y salida con una frecuencia de hasta $5 \mathrm{MHz}$, y cuenta con cuatro contadores que pueden configurarse en tipo $A, B, C$ y $Z$ dependiendo de las necesidades del sistema y el tipo de sensor que se utilice; a su vez, también se pueden configurar las entradas y su modo de operación (i.e. la forma en la que afectan al registro del contador), pudiendo ser dirección, entrada de reloj, "A Quad B", incremento o decremento. La adquisición de las señales digitales se realizará bajo el software Cimplicity Workbench con configuración como sistema tipo SCADA, para adquisición de datos y redistribución al PAC.

\section{Programación en lenguaje Diagrama Escalera}

El primer paso en el desarrollo práctico es la configuración del PAC (Fig. 6), comenzando por la fuente de alimentación IC695CHS012, la CPU o cerebro 
IC695PSD040, el puerto de comunicación y programación que en este caso es bajo el protocolo abierto Ethernet IC695ETM001, un simulador de entradas digitales IC694ACC 300 , salidas digitales IC694MDL754, las entradas rápidas o contadores rápidos IC695HSC304, entradas analógicas IC695ALG600, salidas analógicas IC695ALG704, y un puerto de comunicaciones IC695CMM02.

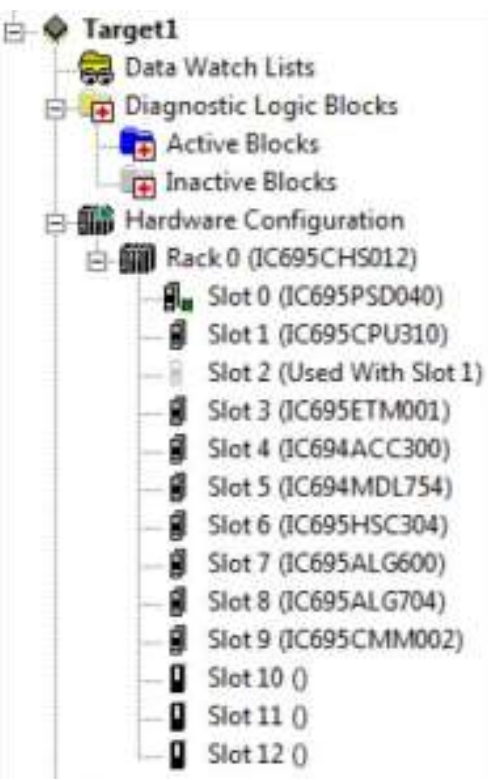

Fig. 6. Menú para la configuración de los diversos módulos a través de los slots del PAC.

Habiendo realizado tal configuración, se continúa configurando la red en la cual estarán enlazados el PAC y la PC en la que se desarrollará la programación y el monitoreo del proceso con la HMI. Se utilizará una red Ethernet bajo el protocolo IPv4 otorgando una dirección de cuatro secciones de ocho bits y una máscara de subred que indica en qué parte de la red se encuentran los dispositivos; mediante la ventana habitual mostrada en la Fig. 7.

Ahí se asignan los parámetros de Dirección IP 10.0.0.X (con posibilidad de conectar hasta 255 dispositivos por estructura de la red), debiendo asignar direcciones diferentes a la PC y al PAC. En este caso particular, el PAC tiene registrada la dirección 10.0.0.8 (Fig. 8), y la PC la 10.0.0.10 (Fig. 7); ambos bajo la misma máscara de subred.

Posteriormente, se prosigue efectuando la creación y conexión del lazo de control; aunado a ello, se realiza la configuración de la conexión entre el PAC y la HMI que se ejecuta en Cimplicity, especificando que el PAC se desempeñará como esclavo y la $\mathrm{HMI}$ como maestro, la cual enviará órdenes y recibirá información que ésta solicite.

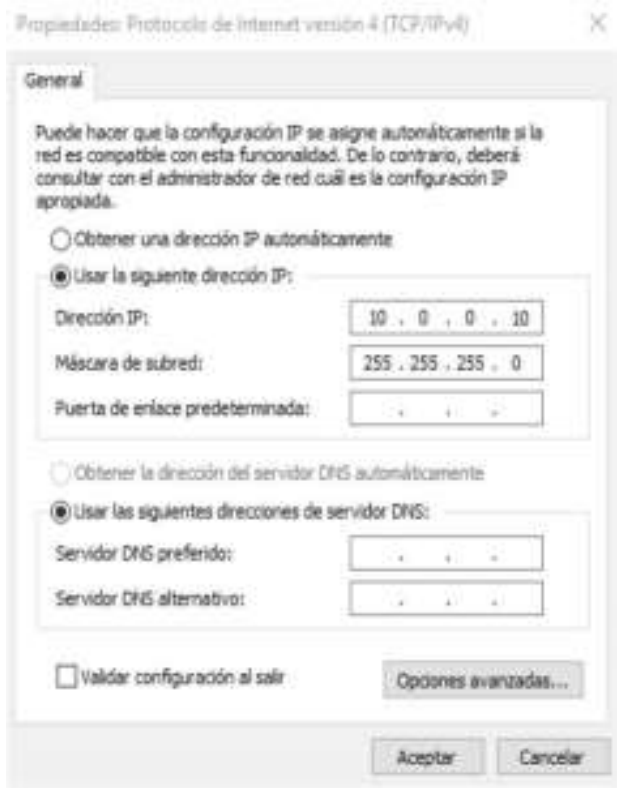

Fig. 7. Ventana de configuración de la red para la comunicación entre la PC y el PAC.

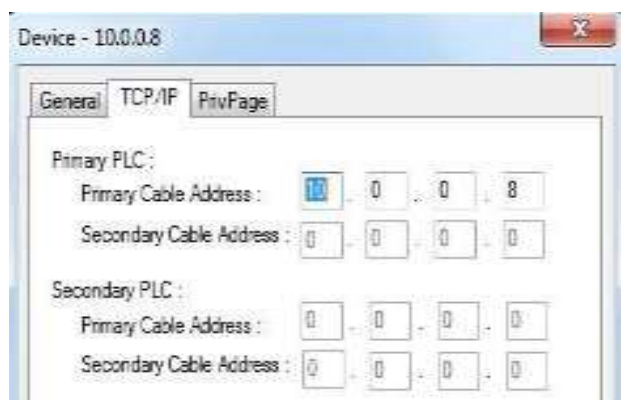

Fig. 8. Ventana de configuración de dirección IP del PAC.

La sincronización se lleva a cabo de manera similar; creando un nuevo proyecto donde se selecciona el protocolo de comunicación. En la sección Ports del menú del proyecto, en el apartado TCP/IP se elige la red que ocupará la $\mathrm{HMI}$, que es la de la PC; y en la pestaña General se agrega el protocolo S90_TRIPLEX; Fig. 9.
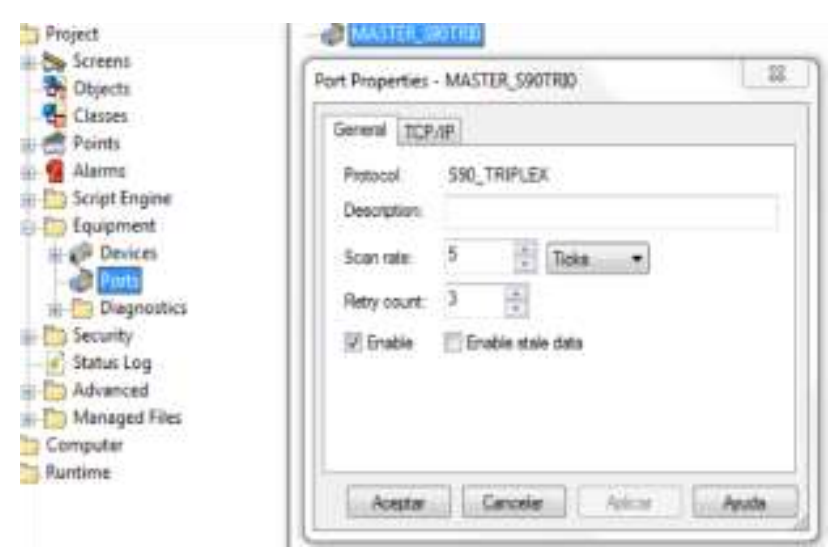

Fig. 9. Configuración de propiedades de puerto y protocolo para la comunicación de la HMI (master). 
Se continúa con la creación de puntos (ya sea de tipo BOOL ó ANALOGIC) necesarios; en este caso se usan cuatro tipos de datos asociados a entradas digitales $(\% \#)$, salidas digitales (\%Q\#), entradas analógicas (\%|A\#), y memorias del sistema (\%M\#). La Fig. 10 muestra los puntos creados para su uso en la HMI.

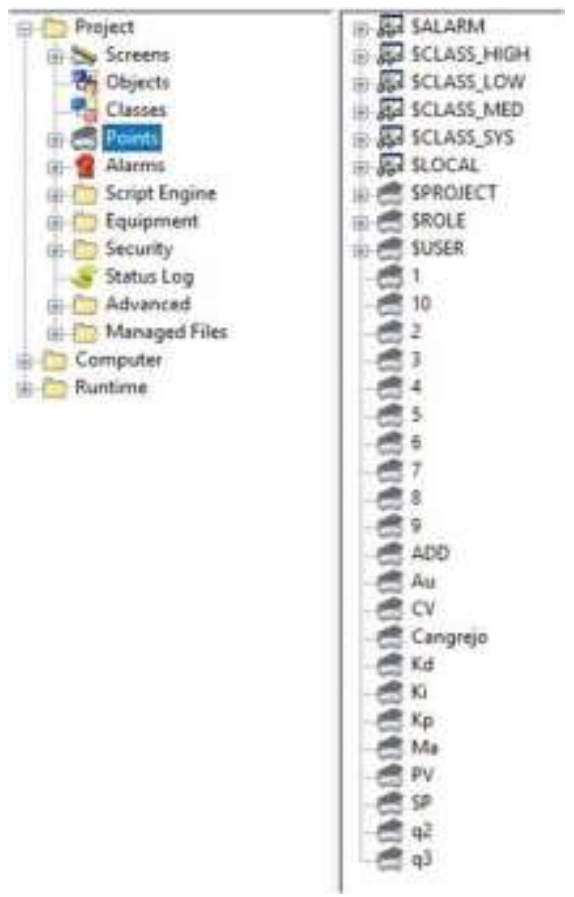

Fig. 10. Puntos creados para su uso en la HMI.

Éstos se relacionan directamente con las variables que se tienen en el PAC, mostradas en la Fig. 11.

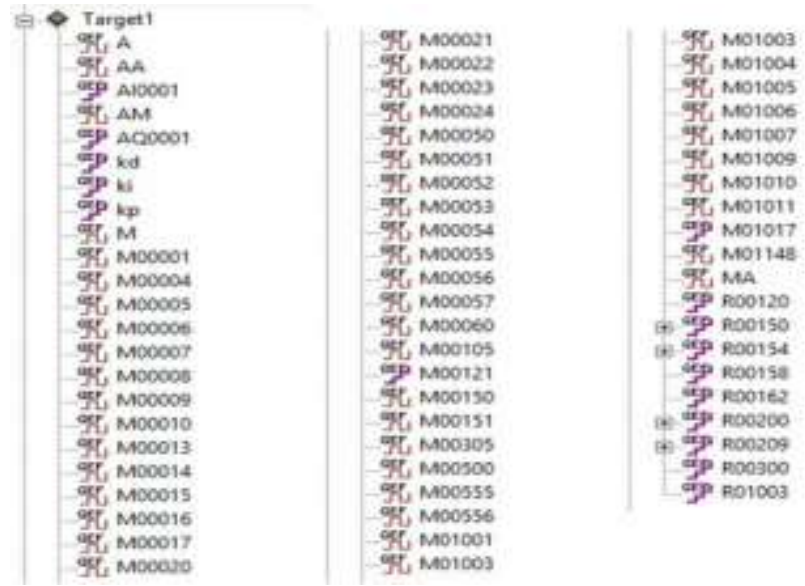

Fig. 11. Variables en el PAC.

En seguida, se realiza la creación y el diseño de Screens.

Y se desarrollan de manera simultánea el lazo de control y la programación de la HMI.

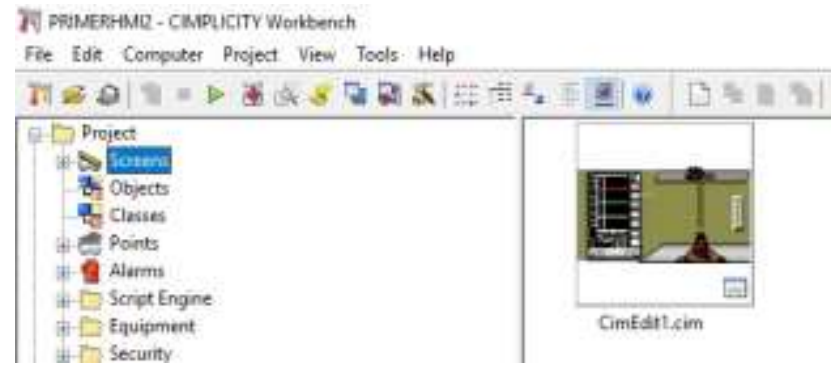

Fig. 12. Ventana para la creación y el diseño de Screens.

\section{HMI}

Su desarrollo se lleva a cabo mediante eventos o acciones creadas o pregrabadas con dibujos u objetos ya existentes, obtenidos mediante el menú mostrado en la Fig. 13; por ejemplo, los presentados en la Fig. 14.

\begin{tabular}{|l|c|}
\hline Picture & A Text Button \\
EObject Explorer & T Text String \\
\hline Q Class Object & \\
\hline Tllustrations & Text \\
\hline
\end{tabular}

Fig. 13. Menú Illustrations > Object Explorer para la construcción de HMIs.

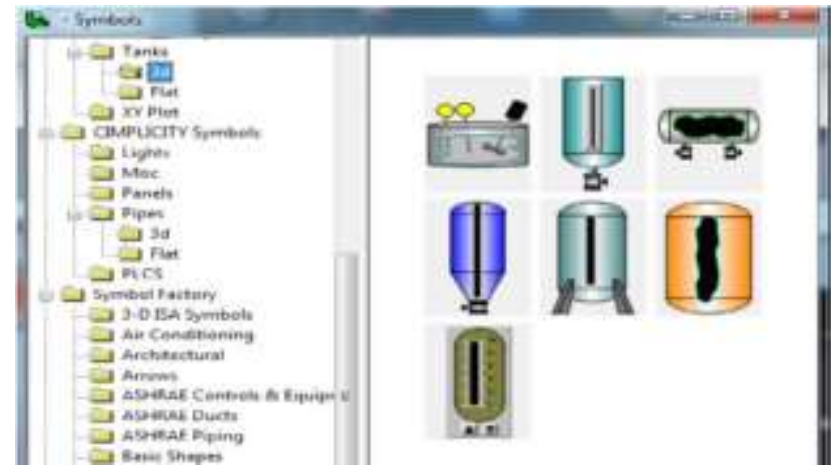

Fig. 14. Objetos prediseñados para la creación de HMls.

Si es necesario o deseado realizar nuevos o diferentes tipos de elementos, se pueden crear y utilizar en la lógica de programación. Una vista general del diseño final realizado en este trabajo se puede observar en la Fig. 15.

Se emplearon botones para efectuar acciones con diferentes reglas de funcionamiento, en modo manual (M) y automático (A). La tendencia de las variables se visualiza mediante el eje numérico del lado izquierdo, con sus respectivos indicadores que presentan valores con exactitud decimal. Por su parte, las tres líneas, con su color respectivo, representan, en rojo la variable de control (CV), en verde el punto de ajuste o Set Point (SP), y en blanco la variable de proceso (PV). Los botones de punta de flecha hacia arriba y punta de flecha hacia abajo, permiten aumentar 0 disminuir, respectivamente, el valor del SP. 


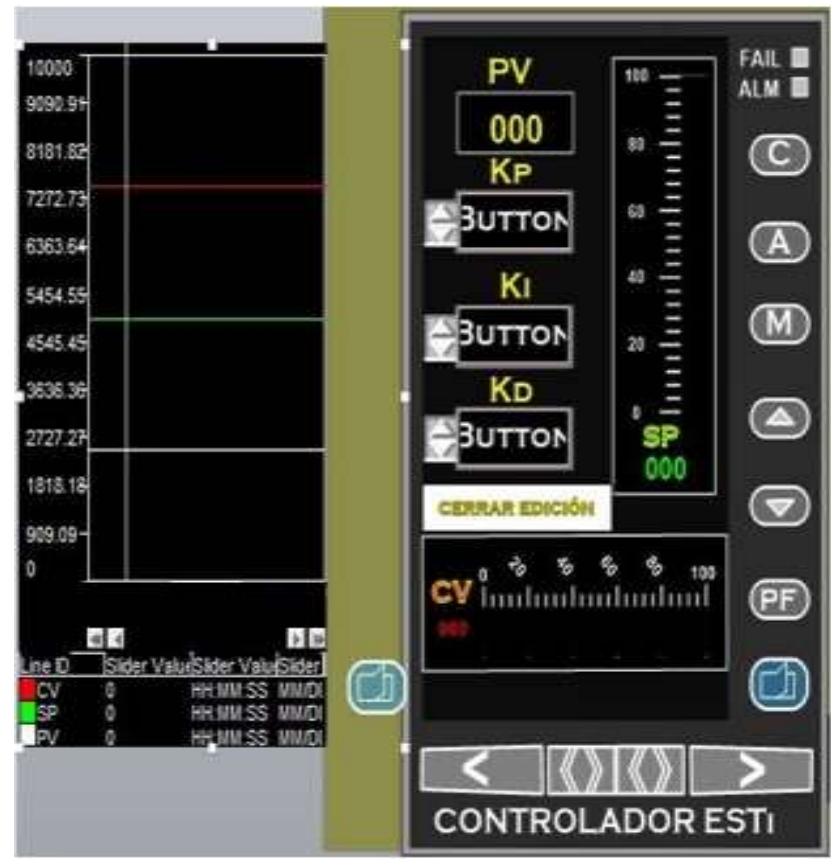

Fig. 15. HMI global del sistema propuesto.

\section{Algoritmo de control}

El lazo de control contiene un bloque denominado PID IND, el cual es un PID industrial con su configuración respectiva. En el código, se realiza el lazo de control bajo el modo de uso, Manual/Automático, implementando timers y contactos. El bloque denominado CALL PID se utiliza para ejecutar el PID desde otro lugar dentro de la lógica en el mismo PAC.

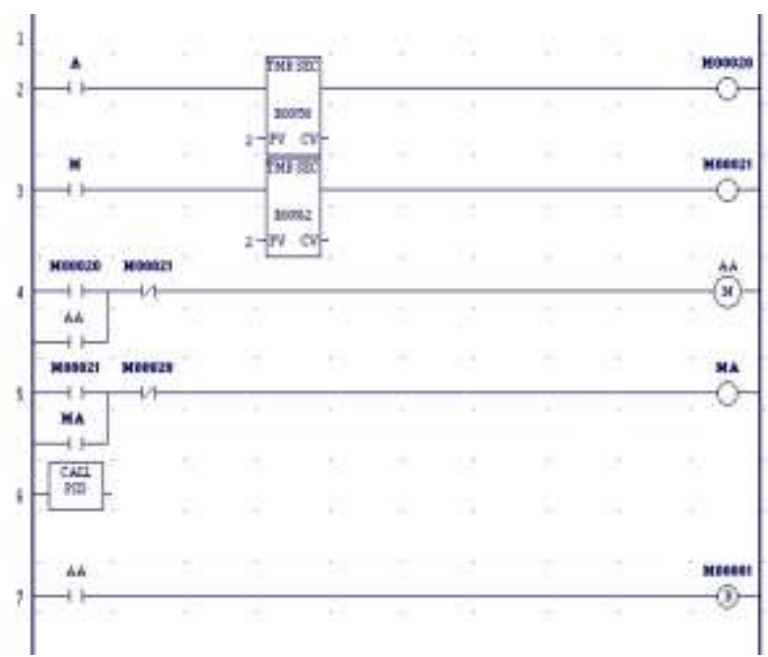

Fig. 16. Programación del algoritmo de control en Diagrama Escalera.

En la lógica se incluye un "movedor" de valores base para el funcionamiento del PID, la banda muerta, el tiempo de muestreo, limites, vías, y las ganancias del controlador. Tales campos se encuentran en la información adicionada al bloque PID. Asimismo, los "contenedores" de variables del modo manual que evitan que se sobrepasen valores mínimos y máximos en sus contadores y límites.

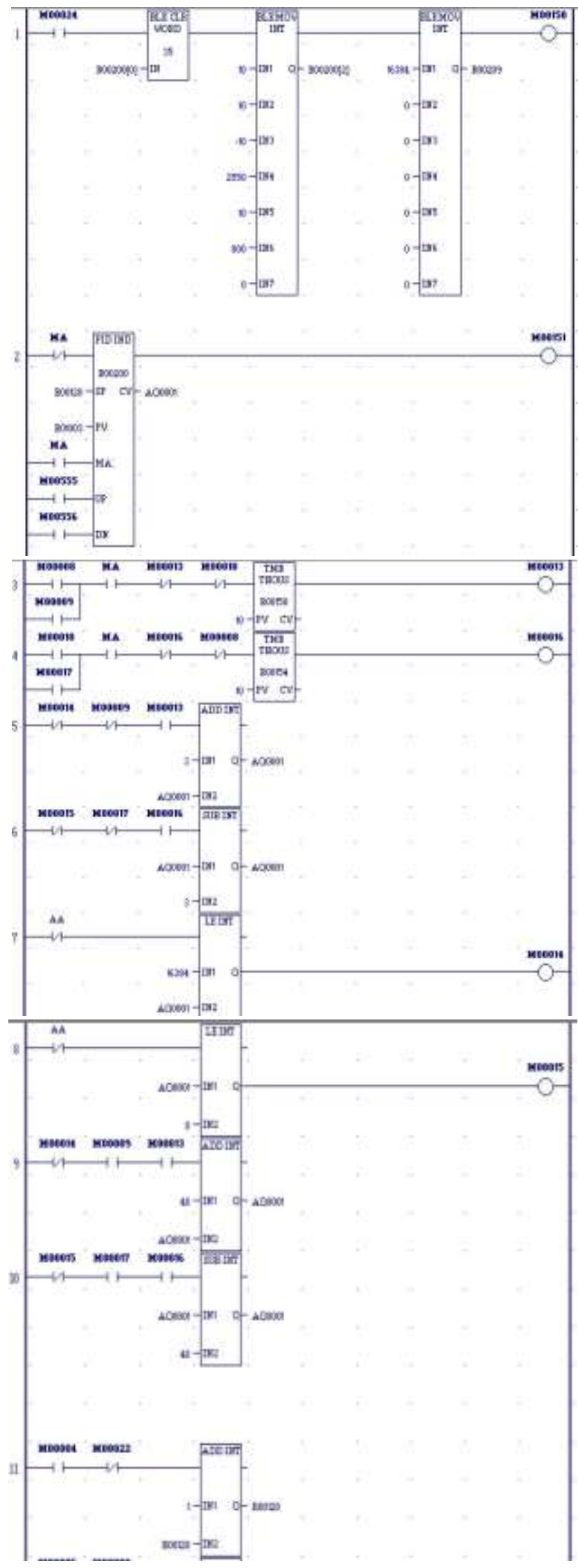




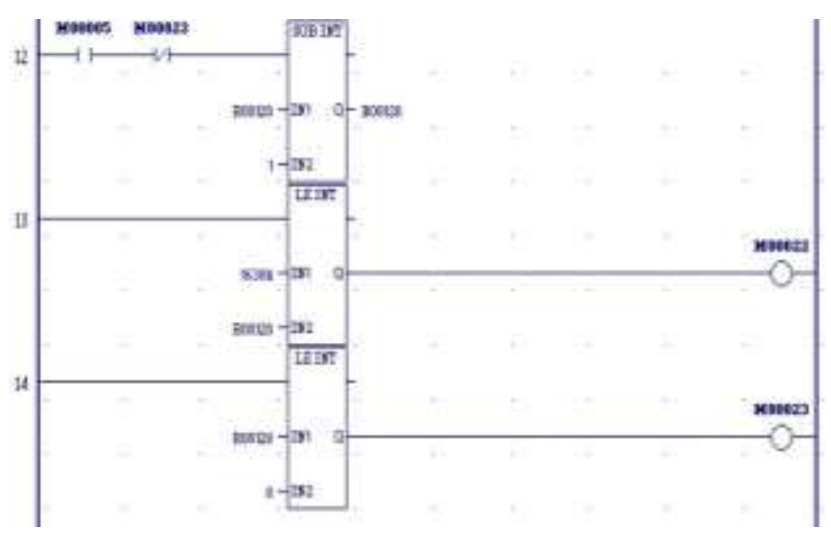

Fig. 16a. Continuación de programación del algoritmo de control.

\section{Conclusiones}

El control de motores en paralelo parece significar una alternativa satisfactoria para la operación de sistemas del tipo de las grúas industriales empleadas para la manipulación de cargas, contribuyendo a la mejora del desempeño y rendimiento de ese tipo de sistemas y a la optimización de recursos como energía, y tiempos y costos de operación, lo que favorece la rentabilidad de las empresas que las utilizan en sus procesos. Este trabajo constituye un proyecto académico terminal de aplicación práctica con enfoque a la automatización de procesos productivos, empleando tecnología de gama industrial, como son los PLC/PAC, las HMI, las redes de comunicación bajo protocolos industriales, los instrumentos de medición, y los actuadores; como propuesta de alternativa de solución a la problemática diversa que se presenta en el campo de acción del Ingeniero en Automatización Industrial, en el desarrollo de los procesos.

\section{Referencias}

[1] A. B. M. G. J.M. Andújar, «Revista Iberoamericana de Automática e Informática Industrial RIAI,» 2 Abril 2007. [En línea]. Available: https://doi.org/10.1016/S1697-7912(07)70212-1.

[2] A. F. Villaverde, «XXVII Jornadas de Automática,» 2006. [En línea]. Available:

https://www.researchgate.net/profile/Alejandro_Villaverde/public ation/264036936_CONTROL DE_UNA_GRUA_PORTICO ME DIANTE_REDES_NEURONALES/links/02e7e53c8fce 03055800 0000.pdf.

[3] J. V. García, «Ingeteam Industry, Parque Tecnológico de Zamudio (Vizcaya),» [En línea]. Available: https:/intranet.ceautomatica.es/old/actividades/jornadas/XXIX/pd f/255.pdf.

[4] A. Anuchin, V. Astakhova, D. Shpak, A. Zharkov y F. Briz, «Optimized method for speed estimation using incremental encoder,» Novi Sad, Serbia , 2017.
[5] West Instruments México, Manual de aplicación de encoders, Ciudad de México, 2009.

[6] S. A. C. Guiraldo, «Control automático educación,» 1 Octubre 2019. [En línea]. Available: https://controlautomaticoeducacion.com/controlrealimentado/control-en-cascada/. [Último acceso: 5 Octubre 2019].

[7] GE Fanuc, «IC695HSC304-DA and IC695HSC308-DA High Speed Counter Modules,» Ge Fanuc, USA, 2013.

[8] GE Intelligent, High-Speed Counter Modules form PACSystem RX3i an 90-30 Series, GE Intelligent Platforms, 2015. 